\title{
Lawson-Norm Based Adaptive Filter for Channel Estimation and in-Car Echo Cancellation
}

This paper was downloaded from TechRxiv (https://www.techrxiv.org).

\section{LICENSE}

CC BY 4.0

SUBMISSION DATE / POSTED DATE

$13-01-2022$ / 19-01-2022

CITATION

Li, Yingsong (2022): Lawson-Norm Based Adaptive Filter for Channel Estimation and in-Car Echo Cancellation. TechRxiv. Preprint. https://doi.org/10.36227/techrxiv.18319319.v1

$\mathrm{DOI}$

10.36227/techrxiv.18319319.v1 


\title{
Lawson-Norm Based Adaptive Filter for Channel Estimation and in-Car Echo Cancellation
}

\author{
Xinqi Huang, Yingsong Li, Senior Member, IEEE, Xiao Han, Huawei Tu
}

\begin{abstract}
We proposed a Lawson-norm-based adaptive estimation algorithm within the affine-project theory framework and give a name of Lawson-norm adaptive filter (LNAF) algorithm. The LNAF algorithm is derived, analyzed, and simulated for echo cancellation when background noise is impulsive, which is realized and implemented via using Lawson norm of past errors to take a sliding window on cost function to speed up convergence and achieve robustness for impulsive noises for colored signal. Simulations based on measured data are used for in-car echo cancellation and channel estimation to verify the LNAF algorithm's performance with different inputs, which prove that the LNAF algorithm is superior to the popular AP algorithms under differnt impulsive interferences.
\end{abstract}

Index Terms-Adaptive filtering, channel estimation, impulsive noise, Lawson norm.

\section{INTRODUCTION}

$\mathbf{T}$ $\mathrm{HE}$ echo is experienced in numerous transmission systems such as vehicle information and video teleconferencing strategies [1]-[3], which will decline the quality of voice transmissions. Adaptive filtering (AF), which is commonly employed for noise reductions in varied situations and has been paid more concerns in recent decades, is an effective method to exclude ambient noises [4]-[9]. The weight updating equations are important for AF algorithms, which are obtained from the derivation of various cost functions constructed using different error criterions. One of the famous AF algorithms is least-mean-square (LMS) that is generally considered for channel equalizations and estimations, noise reductions, and echo cancellations owing to its simplicity under Gaussian assumptions and easy understanding for engineering applications [6]. To make the LMS more robust and increase its behaviors, a normalized LMS (NLMS) and its variants [6], [7], [10] have been devised, analyzed, and discussed for achieving better performance. However, highlycorrelated system inputs such as voice signals will degrade LMS and NLMS algorithms' performance and disturb their behaviors of the strategies. To overcome such drawbacks, affine-projection (AP) theory and its variants [11]-[14] were constructed and considered to improve filters' performance for colored input signals in engineering developments. Nevertheless, these aforementioned algorithms suffer from performance

Xinqi Huang and Yingsong $\mathrm{Li}$ are with the College of Information and Communication Engineering, Harbin Engineering University, Harbin 150001, China. (e-mail: liyingsong@ieee.org).

Xiao Han is with the College of Computer Science and Technology, Harbin Engineering University, Harbin 150001, China.

Huawei Tu is with the Department of Computer Science and Information Technology, La Trobe University, Melbourne, VIC 3086, Australia (e-mail: h.tu@latrobe.edu.au) deterioration as the background system noises are heavy-tail distributed impulsive noises [15]-[21].

To speed up the convergence of these early devised AF algorithms for various impulsive noise applications, an affineprojection-sign (APS) algorithm [22] was developed, which utilizes $l_{1}$-norm to minimize a posteriori error vector. Since matrix inversion for APS is not needed, the APS's weight updating equation is to simplify the computations. Additionally, it augments the convergence in comparison with the basic AP algorithm when we deal with impulsive interferences. To further enhance its performance, an affine-projection-Versoria (APV) algorithm was reported in [2], which introduces datareusing into Versoria-cost function to improve filter performance. With the benefit combination of the above superiorities, the APV algorithm gains stability for suppressing large outliers and accelerates convergence with correlated signal inputs.

This brief brings a Lawson-norm promoted AF (LNAF) algorithm to further enhance AP's convergence and steadystate performance if the system encounters impulsive interferences, and is robust for correlated inputs, and reduces effects from large outliers. Similar to the Lorentzian adaptive filtering (LAF) algorithm [23], motivated by the AP scheme and Lawson-norm [24]-[26], the constructed LNAF algorithm is obtained through taking gradient descent search on a Lawson norm promoting cost function to ensure robustness. The learning rate boundary for achieving stable convergence of the LNAF algorithm is presented and driven. The complexity of the LNAF algorithm is also presented and compared with existing schemes. Simulation results for vehicle based echochannel-estimations and echo cancellations demonstrate that the behaviors of devised LNAF algorithm are better than the AP, APV, APS algorithms under impulsive system interferences.

\section{Proposed LNAF AlgorithM}

Assume that an unknown system has an output

$$
d(n)=\mathrm{y}(n)+v(n),
$$

where $v(n)$ is regarded as background noise whose variance at time index $n$ is $\sigma_{v}^{2}$, and $\mathrm{y}(n)=\mathbf{x}^{T}(n) \mathbf{w}_{\mathrm{o}}$ with $\mathbf{x}(n)=$ $[x(n), x(n-1), \ldots, x(n-N+1)]^{T}$ is system input signal vector whose covariance matrix is $\mathbf{R}_{x}=E\left[\mathbf{x}(n) \mathbf{x}^{T}(n)\right]$, $\mathbf{w}_{\mathrm{o}} \in \mathbf{R}^{N \times 1}$ is an unknown vector to be estimated, and $(\cdot)^{T}$ represents the transpose operator. The error vector $\mathbf{e}(n)=[e(n), e(n-1), \ldots, e(n-M+1)]^{T}$ for $\mathrm{AF}$ with weight vector $\mathbf{w}(n)$ of estimated $\mathbf{w}_{\mathrm{O}}$ is obtained and presented 
as

$$
\mathbf{e}(n)=\mathbf{d}(n)-\mathbf{X}_{n}^{T} \mathbf{w}(n) .
$$

In this case, $\mathbf{X}_{n}=[\mathbf{x}(n), \mathbf{x}(n-1), \ldots, \mathbf{x}(n-M+1)]$ and $\mathbf{d}(n)=[d(n), d(n-1), \ldots, d(n-M+1)]^{T}$ have projection order $M$ to reuse data for combating outlier interferences.

As we know, the APS algorithm updates its weight vector utilizing sign information of error signal when the system runs into impulsive environments. Like the LAF algorithm, the LNAF algorithm is developed uses a Lawson norm [24], [25] to take full advantage of the error information to improve system performance for impulsive scenarios. To construct a robust cost function for LNAF algorithm, the Lawson-norm function integrated with AP scheme [2], [23] is used and given by

$$
J_{l n}(n)=\sum_{i=0}^{M-1} \frac{e^{2}(n-i)}{\left(e^{2}(n-i)+\lambda^{2}\right)^{\frac{2-p}{2}}},
$$

where $0 \leq p \leq 2$ is a norm adjusting parameter to control an approximation of $l_{p}$ norm, and $\lambda$ is a sufficiently small parameter for regularization. Figure 1 gives the effects of parameter $p$ on $J_{l n}(n)$ with $M=1$ and $\lambda=0.7$. As $p$ increases, the large element of $e(n)$ is put larger weight. In the minimization process, the large element will be penalized strongly.

Taking partial derivative on (3) with respect to $\mathbf{w}(n)$, yields

$$
\begin{aligned}
\frac{\partial J_{l n}(n)}{\partial \mathbf{w}(n)} & =\frac{\partial}{\partial \mathbf{w}(n)} \sum_{i=0}^{M-1} \frac{e^{2}(n-i)}{\left(e^{2}(n-i)+\lambda^{2}\right)^{\frac{2-p}{2}}} \\
& =-\mathbf{X}_{n} \mathbf{H}_{n} \mathbf{e}(n),
\end{aligned}
$$

where $\mathbf{H}_{n}=\operatorname{diag}\left[h_{1}(n), h_{2}(n), \cdots, h_{M}(n)\right]$ is a $M \times M$ having diagonal elements of

$$
h_{k}(n)=\frac{\left(2 \lambda^{2}+p e^{2}(n-k+1)\right)}{\left(e^{2}(n-k+1)+\lambda^{2}\right)^{\frac{4-p}{2}}}, k=1,2, \ldots, M .
$$

From the constructed cost function in (3), the LNAF algorithm is derived using gradient-descent method (GDM) [25], and finally, we get weight updating equation

$$
\mathbf{w}(n+1)=\mathbf{w}(n)-\mu \frac{\partial}{\partial \mathbf{w}(n)}\left(J_{l n}(n)\right),
$$

where $\mu$ is regarded as a step size of the LNAF algorithm to provide a balance between convergence and misalignment. Using equations (4) and (6), yields

$$
\mathbf{w}(n+1)=\mathbf{w}(n)+\mu \mathbf{X}_{n} \mathbf{H}_{n} \mathbf{e}(n) .
$$

The robustness of the LNAF algorithm for combating impulsive interferences is reasoned from the matrix whose behavior

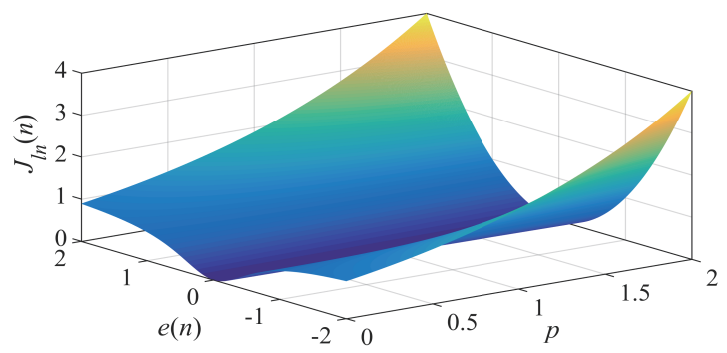

Fig. 1: The Lawson-norm function in $3 \mathrm{D}$ space with $M=1$ and $\lambda=0.7$.

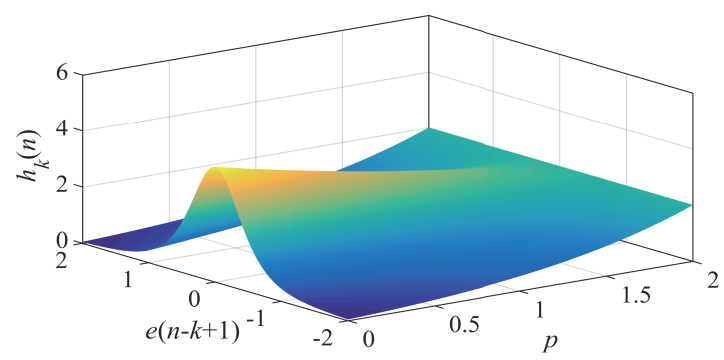

Fig. 2: The $h_{k}(n)$ in 3D space with $\lambda=0.7$.

is presented in Fig. 2. Here, a stronger penalty is exerted on large errors, while a weaker penalty is put on small errors with same $p$ to remove the adverse impact of the impulsive noises. As $p$ decreases, the LNAF algorithm is more robust since it is less sensitive to outliers.

\section{StabiLity ANALYSIS}

To better understand the devised LNAF algorithm, its stability analysis is presented to get the boundary of learning rate, where the energy conversation method is considered, which has been used for AF analysis for several decades [12], [26]. Let $\tilde{\mathbf{w}}(n)=\mathbf{w}_{\mathrm{o}}-\mathbf{w}(n)$, yields

$$
\begin{aligned}
\tilde{\mathbf{w}}(n+1) & =\mathbf{w}_{\mathrm{o}}-\mathbf{w}(n+1) \\
& =\tilde{\mathbf{w}}(n)-\mu \mathbf{X}_{n} \mathbf{H}_{n} \mathbf{e}(n) \\
& =\tilde{\mathbf{w}}(n)-\mu \mathbf{X}_{n} \mathbf{H}_{n}\left[\mathbf{X}_{n}^{T} \mathbf{w}_{\mathrm{o}}+\mathbf{v}(n)-\mathbf{X}_{n}^{T} \mathbf{w}(n)\right] \\
& =\tilde{\mathbf{w}}(n)-\mu \mathbf{X}_{n} \mathbf{H}_{n}\left[\mathbf{X}_{n}^{T} \tilde{\mathbf{w}}(n)+\mathbf{v}(n)\right] \\
& =\left[\mathbf{I}-\mu \mathbf{X}_{n} \mathbf{H}_{n} \mathbf{X}_{n}^{T}\right] \tilde{\mathbf{w}}(n)-\mu \mathbf{X}_{n} \mathbf{H}_{n} \mathbf{v}(n),
\end{aligned}
$$

where $\mathbf{v}(n)=[v(n), v(n-1), \ldots, v(n-M+1)]^{T}$.

Assuming that $v(n)$ denotes zero-mean (ZM) independent, identically, distributed (i.i.d.) noise and is independent of any other signals, and $\tilde{\mathbf{w}}(n)$ is statistical independent with $\mathbf{X}_{n} \mathbf{H}_{n} \mathbf{X}_{n}^{T}$. Then, we get

$$
\begin{aligned}
E[\tilde{\mathbf{w}}(n+1)] & =E\left[\mathbf{I}-\mu \mathbf{X}_{n} \mathbf{H}_{n} \mathbf{X}_{n}^{T}\right] E[\tilde{\mathbf{w}}(n)] \\
& =\left\{\mathbf{I}-\mu E\left[\mathbf{X}_{n} \mathbf{H}_{n} \mathbf{X}_{n}^{T}\right]\right\} E[\tilde{\mathbf{w}}(n)] .
\end{aligned}
$$

To make algorithm converge, equation (9) should satisfy

$$
\left|1-\mu \lambda_{\max }\left(E\left[\mathbf{X}_{n} \mathbf{H}_{n} \mathbf{X}_{n}^{T}\right]\right)\right|<1
$$

where $\lambda_{\max }\left(E\left[\mathbf{X}_{n} \mathbf{H}_{n} \mathbf{X}_{n}^{T}\right]\right)$ is the maximum eigenvalue of matrix $E\left[\mathbf{X}_{n} \mathbf{H}_{n} \mathbf{X}_{n}^{T}\right]$. After calculations, equation (10) is changed to

$$
0<\mu<\frac{2}{\lambda_{\max }\left(E\left[\mathbf{X}_{n} \mathbf{H}_{n} \mathbf{X}_{n}^{T}\right]\right)} .
$$

Assuming independence between $\mathbf{X}_{n} \mathbf{X}_{n}^{T}$ and $\mathbf{H}_{n}$ [23], yields

$$
\begin{aligned}
\lambda_{\max }\left(E\left[\mathbf{X}_{n} \mathbf{H}_{n} \mathbf{X}_{n}^{T}\right]\right) & =\lambda_{\max }\left(E\left[\mathbf{X}_{n} E\left[\mathbf{H}_{n}\right] \mathbf{X}_{n}^{T}\right]\right) \\
& =\lambda_{\max }\left(\operatorname{Tr}\left\{E\left[\mathbf{H}_{n}\right]\right\} \mathbf{R}_{x}\right) .
\end{aligned}
$$

Then, taking derivative on $h_{k}(n)$ with respect to $e(n-k+1)$, yields

$$
h_{k}^{\prime}(n)=\frac{\left[\left(p^{2}-2 p\right) e^{2}(n-k+1)+4 \lambda^{2}(p-2)\right] e(n-k+1)}{\left(e^{2}(n-k+1)+\lambda^{2}\right)^{\frac{6-p}{2}}}
$$

Making formula (13) to be zero, we can obtain that $e(n-k+1)=0$ is the extreme point when $p \neq 2$. From the Fig. 2, $e(n-k+1)=0$ is the maximum point. As a 
result, the maximum of $h_{k}(n)$ is received

$$
\left\{\begin{array}{cc}
h_{k}(n) \leq 2 \lambda^{p-2} & p \neq 2 \\
h_{k}(n)=2 & p=2
\end{array} .\right.
$$

Since $\lambda^{p-2}$ decreases as $p$ increases and $\lambda$ is a small positive parameter, we can procure that $\lambda^{p-2} \leq \lambda^{-2}$ and $\lambda^{-2}>1$. Then, we obtain

$$
h_{k}(n) \leq 2 \lambda^{-2} .
$$

After calculations, equation (12) is changed to

$$
\lambda_{\max }\left(E\left[\mathbf{X}_{n} \mathbf{H}_{n} \mathbf{X}_{n}^{T}\right]\right)<2 M \lambda^{-2} \lambda_{\max }\left(\mathbf{R}_{x}\right) .
$$

Then, the boundary can be achieved from equation (11), which can be further written as

$$
0<\mu<\frac{\lambda^{2}}{M \lambda_{\max }\left(\mathbf{R}_{x}\right)} .
$$

\section{Computational Complexity}

Table 1 presents the computational complexity for the proposed LNAF algorithm compared with the AP, APV, LAF, and APS regarding the total number of multiplications, additions, and divisions. Computational complexity of the AP algorithm is larger because it requires more matrix inversion.

TABLE I: Complexity analysis of mentioned algorithms

\begin{tabular}{cccc}
\hline Algorithm & Multiplication & Division & Addition \\
\hline AP & $\left(M^{2}+2 M\right) N+M^{3}+M$ & $-\left(M^{2}+2 M\right) N+M^{3}+M^{2}-M$ \\
APS & $2 M N+M^{2}+4 M$ & 1 & $2 M N+M^{2}+2 M-1$ \\
APV & $M N+N+2 M^{2}+6 M+2$ & $M+1$ & $M N+N+2 M M^{2}+4 M$ \\
LAF & $2 M N+3 M$ & $M$ & $2 M N+M$ \\
LNAF & $2 M N+4 M$ & $M$ & $2 M N+2 M$ \\
\hline
\end{tabular}

\section{Simulation RESUlTS}

Experiments are designed and analyzed to validate the performance of LNAF algorithm via echo cancellations and channel estimations. Here, an acoustic echo canceller (AEC) system and hands-free cell phone (HFCP) framework with single and double talk scenarios are considered. The AP, APV, APS, and LAF algorithms are employed for a sake of comparison. In Case I, how the parameters affect the performance is investigated. In Case II, the behaviors for AP, APV, APS, LAF, and LNAF algorithms are evaluated for AEC application. For Cases III and IV, the AEC scenarios are considered, simulated using measured speech inputs and discussed. The results are gotten via averaging 100 independent trials, where the regularization parameters for AP, APS, and APV algorithms are set to be $10^{-6}$, and all coefficients are initialized to zero.

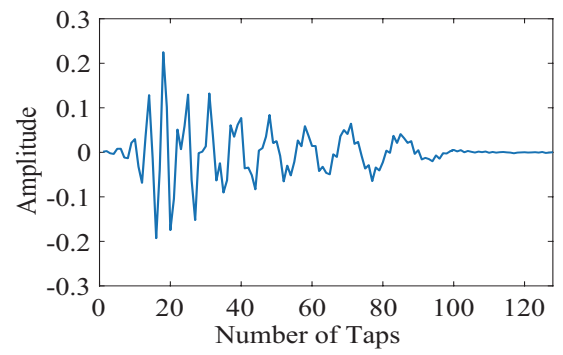

Fig. 3: 128-tap Echo channel impulse response.

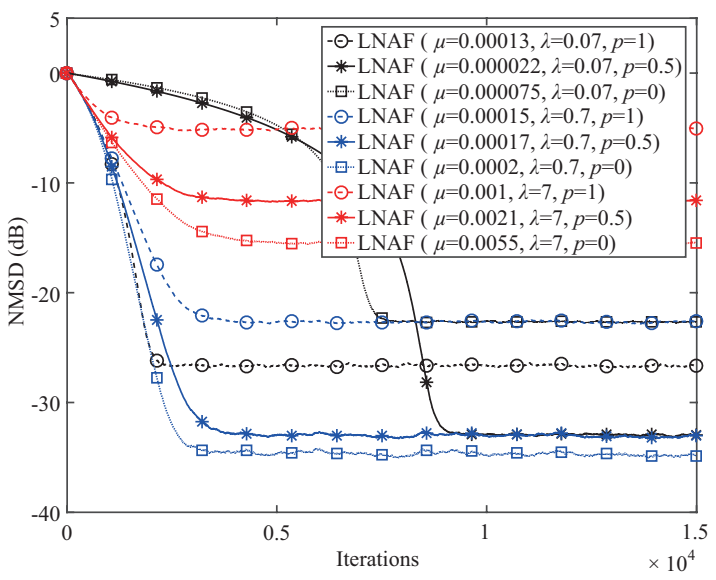

(a)

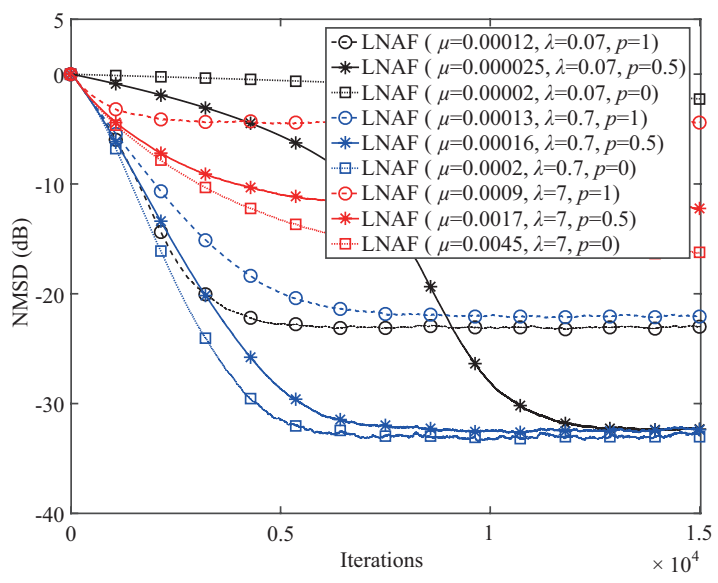

(b)

Fig. 4: NMSD performance of APQR algorithm for different inputs (a) WGN signal and (b) AR signal. (Case I)

\section{A. Echo Channel Estimation}

Here, the unknown system $\mathbf{w}_{\mathbf{o}}$ is a channel model $\mathrm{p}$ resented in Fig. 3, which is to be estimated having a length of 128 for AEC application. The length of AF has same length as the channel. The normalized mean-square deviation (NMSD) is selected for performance metric given by $\operatorname{NMSD}(n)=10 \log _{10}\left[\left\|\mathbf{w}(n)-\mathbf{w}_{\mathrm{o}}\right\|^{2} /\left\|\mathbf{w}_{\mathrm{o}}\right\|^{2}\right]$. White Gaussian noise (WGN) and first-order autoregressive (AR) random process $x(n)=0.9 x(n-1)+u(n)$ are chosen to input the system for comparing the performance of above popular algorithms. Herein, $u(n)$ is a WGN with unit variance and zero-mean. A contaminated non-Gaussian noise model $v(n)=\alpha(n)+b(n) \beta(n)$ [2], [25] is modeled as system background noise. Here, $b(n)$ is an independent identicallydistributed (i.i.d.) Bernoulli random sequence with occurrence probability of $\operatorname{Pr}[b(n)=1]=P$ and $\operatorname{Pr}[b(n)=0]=1-P$. $\alpha(n)$ and $\beta(n)$ are both i.i.d. zero mean WGNs having variances of $\sigma_{\alpha}^{2}$ and $\sigma_{\beta}^{2}\left(\sigma_{\beta}^{2} \gg \sigma_{\alpha}^{2}\right)$, respectively.

In Case I, the convergence of the LNAF with different $\lambda$ and $p$ are to investigate parameter effect on LNAF's performance. $v(n)$ disturbs the system with $P=0.1$, where a signal-to-interference ratio (SIR) of $-30 \mathrm{~dB}$ and a signalto-noise ratio (SNR) of $30 \mathrm{~dB}$ are used. The SIR is real- 


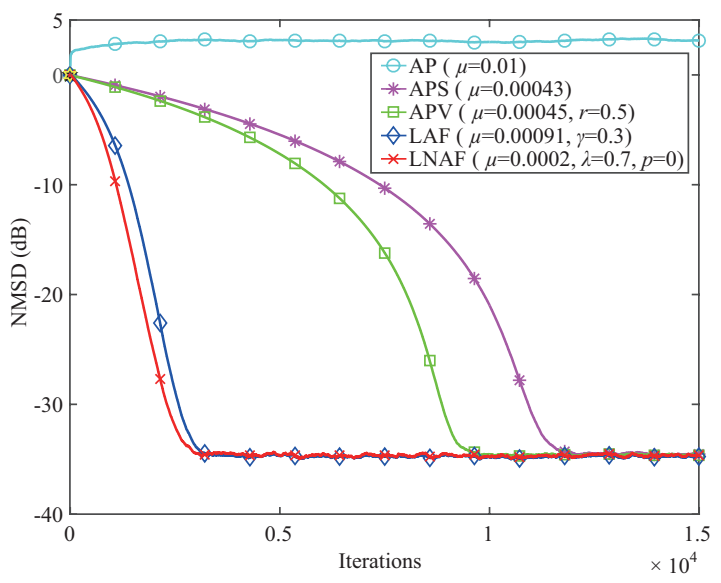

(a)

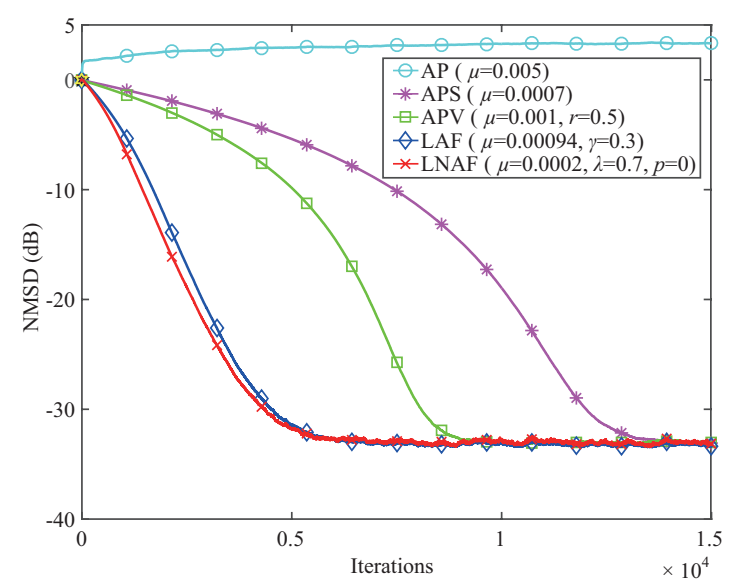

(b)

Fig. 5: NMSD performance of AP, APS, APV, LAF and LNAF algorithms for the input signals (a) WGN signal and (b) AR signal. (Case II)

ized using $\mathrm{SIR}=10 \log _{10}\left\{E\left[\mathrm{y}^{2}(n)\right] / \sigma_{\beta}^{2}\right\}$, while the SNR is $\mathrm{SNR}=10 \log _{10}\left\{E\left[\mathrm{y}^{2}(n)\right] / \sigma_{\alpha}^{2}\right\}$, and $M=4$. The parameter $\mu$ is listed in the Figs. 4. We can conclude that when $\lambda$ is too small or too large, LNAF's performance becomes worse for WGN and AR input signals, and the LNAF algorithm achieves the best performance for $\lambda=0.7$ and $p=0$.

In Case II, the AP, APV, APS, LAF, and LNAF algorithms are taken for investigating different input signals with the same noise model mentioned before. Figures 5 illustrates that all algorithms can work well under impulsive environment with WGN and AR inputs except AP algorithm, while the LNAF algorithm has faster convergence than other compared algorithms for the same steady-state NMSD. It is noted that

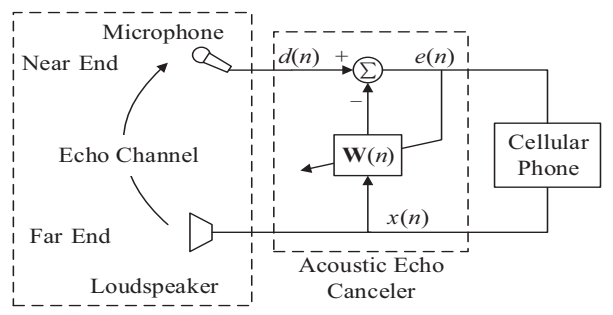

Fig. 6: Diagram of hands-free cellular phone inside an car.

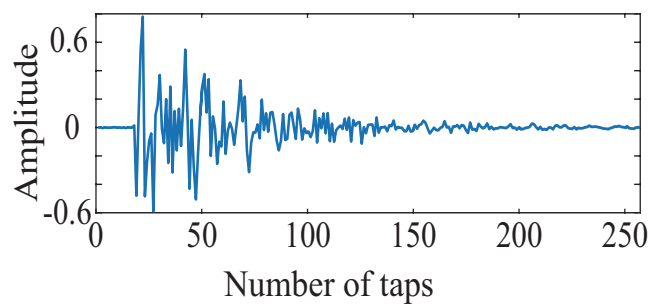

Fig. 7: In-car echo path impulse response with 256 taps.

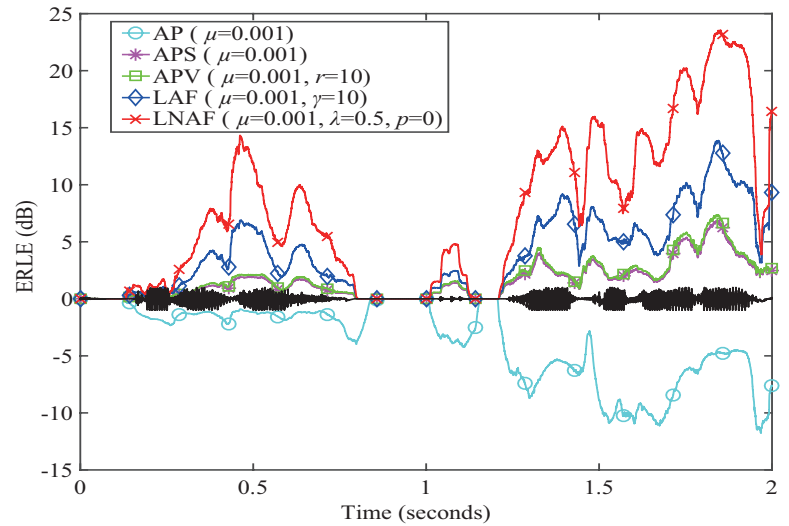

Fig. 8: ERLE Comparison of these algorithms for single-talk scenario. (Case III)

the LNAF's performance is slightly better than the LAF for AR input.

\section{B. Echo Cancellation}

Then, the HFCP framework [27]-[29] with double-talk and single-talk scenarios are created and discussed for comparing above algorithms. An illustration of the HFCP block diagram is presented in Fig. 6, and a 256-tap in-car echo channel in a normal automobile is employed [29] and shown in Fig. 7. The AF length is same as in-car echo channel, and the echo-return-loss-enhancement (ERLE) is to measure the effectiveness of estimation, having $\operatorname{ERLE}(n)=$ $10 \log _{10}\left(E\left[d^{2}(n)\right] / E\left[e^{2}(n)\right]\right)$. Also, time-averaging is used to estimate expectations [30], and both near-end and far-end authentic speeches are sampled at $8 \mathrm{kHz}$.

In Case III, a single-talk scenario is considered and discussed with far-end signal plotting in Fig. 9, and $M=4$. The parameters SNR, SIR, and $P$ are set to $30 \mathrm{~dB},-30 \mathrm{~dB}$, and 0.001, respectively. The ERLE behaviors are presented in Fig. 8. The proposed LNAF algorithm's performance is better than mentioned algorithms for comparisons.

In Cases IV, a double-talk scenario is constructed and investigated, while parameters are same as Case III. Ad-
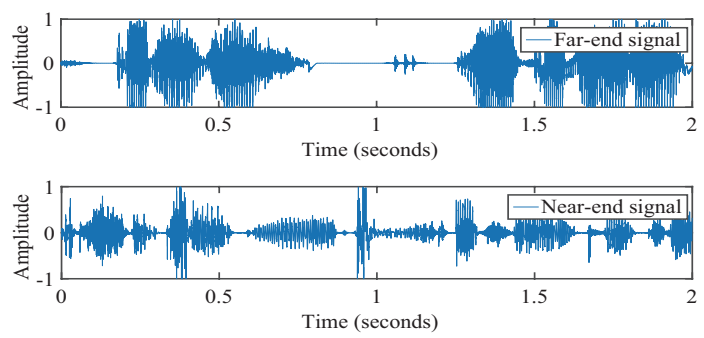

Fig. 9: The near-end and far-end speech signals for double-talk scenario. (Case IV) 


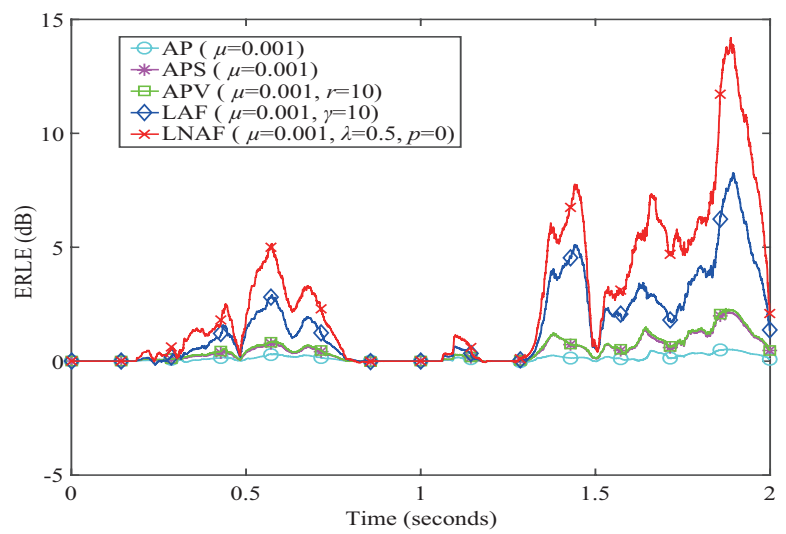

Fig. 10: ERLE Comparison of these algorithms for double-talk scenario. (Case IV)

ditionally, the Geigel double-talk detection is used for restraining weight vector adaptation of the filters if $|d(n)| \geq$ $1 / 2 \max \{|x(n)|,|x(n-1)|, \ldots|x(n-N+1)|\}$ [31]. As can be seen from Fig. 10, the LNAF algorithm shows superior performance compared with other employed algorithms.

\section{CONCLUSIONS}

In this brief, the Lawson-norm adaptive filter (LNAF) algorithm has been proposed, analyzed, and discussed to provide faster convergence and reduce noise interference effects for channel estimation within the robust adaptive filtering designs. The LNAF algorithm is realized by taking a sliding window on a Lawson norm constructed cost function with past errors against impulsive noise. Simulation results illustrate that the LNAF algorithm provides desired convergence behaviors compared to mentioned algorithms for combating impulsive interferences.

\section{REFERENCES}

[1] S. Nordholm, I. Claesson, and B. Bengtsson, "Adaptive array noise suppression of handsfree speaker input in cars," IEEE Trans. Veh. Technol., vol. 42, no. 4, pp. 514-518, Nov. 1993.

[2] F. Huang, J. Zhang, and S. Zhang, "Affine projection Versoria algorithm for robust adaptive echo cancellation in hands-free voice communications," IEEE Trans. Veh. Technol., vol. 67, no. 12, pp. 11924-11935, Dec. 2018.

[3] C.-H. Yang, J.-C.Wang, J.-F.Wang, C.-H.Wu, and K.-H. Chang, "Design and implementation of subspace-based speech enhancement under incar noisy environments," IEEE Trans. Veh. Technol., vol. 57, no. 3, pp. 1466-1479, May 2008.

[4] T. Liang, Y. Li, W. Xue, Y. Li, T. Jiang, "Performance and Analysis of Recursive Constrained Least Lncosh Algorithm under Impulsive Noises," IEEE Trans. Circuits Syst. II, Express Briefs, vol. 68, no. 6, pp. 2217-2221, Nov. 2020.

[5] Y. Li, Y. Wang, and T. Jiang, "Sparse-aware set-membership NLMS algorithms and their application for sparse channel estimation and echo cancelation," AEU-Int. J.Electron. Coтmu., vol. 70, no. 7, pp. 895-902, Jul. 2016.

[6] S. Haykin, Adaptive Filter Theory, Upper Saddle River, NJ, USA: Prentice-Hall, 2002.

[7] A. H. Sayed, Fundamentals of Adaptive Filtering. Hoboken, NJ, USA: Wiley, 2003

[8] Z. Zheng, Z. Liu, and Y. Dong, "Steady-state and tracking analyses of the improved proportionate affine projection algorithm," IEEE Trans. Circuits Syst. II, Express Briefs, vol. 65, no. 11, pp. 1793-1797, Nov. 2018.
[9] N. R. Yousef, and A. H. Sayed, "Steady-state and tracking analyses of the sign algorithm without the explicit use of the independence assumption," IEEE Signal Process. Lett., vol. 7, no. 11, pp. 307-309, Nov. 2000.

[10] Y. Li, Y. Wang, and T. Jiang, "Norm-adaption penalized least mean square/fourth algorithm for sparse channel estimation," Signal Process., vol. 128, no. 2016, pp. 243-251, Apr. 2016

[11] K. Ozeki and T. Umeda, "An adaptive filtering algorithm using an orthogonal projection to an affine subspace and its properties," Electron. Commun. Jpn., vol. 67, no. 5, pp. 19-27, May 1984.

[12] H. C. Shin and A. H. Sayed, "Mean-square performance of a family of affine projection algorithms," IEEE Trans. Signal Process., vol. 52, no. 1, pp. 90-102, Jan. 2004.

[13] S. J. Ban, C. W. Lee, and S. W. Kim, "Adaptive regularization parameter for pseudo affine projection algorithm," IEEE Signal Process. Lett., vol. 16, no. 5, pp. 382-385, May 2009.

[14] W. A. Martins, M. V. S. Lima, P. S. R. Diniz, and T. N. Ferreira, "Optimal constraint vectors for set-membership affine projection algorithms," Signal Process., vol. 134, pp. 285-294, Jan. 2017.

[15] M. Z. A. Bhotto, M. O. Ahmad, and M. N. S. Swamy, "Robust shrinkage affine-projection sign adaptive-filtering algorithms for impulsive noise environments," IEEE Trans. Signal Process., vol. 62, no. 13, pp. 33493359, Jul. 2014.

[16] W. Shi, Y. Li, and Y. Wang, "Noise-free maximum correntropy criterion algorithm in non-Gaussian environment," IEEE Trans. Circuits Syst. II, Express Briefs, vol. 67, no. 10, pp. 2224-2228, Oct. 2020.

[17] T. Ogunfunmi and T. Paul, "The quarternion maximum correntropy algorithm," IEEE Trans. Circuits Syst. II, Express Briefs, vol. 62, no. 6, pp. 598-602, Feb. 2015.

[18] B. Chen, L. Xing, H. Zhao, N. Zheng, and J. C. Principe, "Generalized correntropy for robust adaptive filtering," IEEE Trans. Signal Process., vol. 64, no. 13, pp. 3376-3387, Jul. 2016.

[19] Y. Li, Z. Jiang, W. Shi, X. Han, and B. Chen, "Blocked maximum correntropy criterion algorithm for cluster-sparse system identifications," IEEE Trans. Circuits Syst. II, Express Briefs, vol. 66, no. 11, pp. 19151919, Nov. 2019.

[20] F. Huang, J. Zhang, and S. Zhang, "Adaptive filtering under a variable kernel width maximum correntropy criterion," IEEE Trans. Circuits Syst. II, Express Briefs, vol. 64, no. 10, pp. 1247-1251, Oct. 2017.

[21] P. Song and H. Zhao, "Affine-projection-like M-estimate adaptive filter for robust filtering in impulse noise," IEEE Trans. Circuits Syst. II, Express Briefs, vol. 66, no. 12, pp. 2087-2091, Feb 2019.

[22] T. Shao, Y. R. Zheng, and J. Benesty, "An affine projection sign algorithm robust against impulsive interferences," IEEE Signal Process. Lett., vol. 17, no. 4, pp. 327-330, Apr. 2010.

[23] R. L. and M. Narwaria, "Lorentzian Based Adaptive Filters for Impulsive Noise Environments," IEEE Trans. Circuits Syst. I, Regular Papers, vol. 64, no. 6, pp. 1529-1539, Jun. 2017.

[24] D. Fournier and D. W. Oldenburg, "Inversion using spatially variable mixed $l_{p}$ norms," Geophys. J. Int., vol. 218, no. 1, pp. 268-282, May 2019.

[25] S. Vatankhah, R. A. Renaut, and V. E. Ardestani, "3-D projected $L_{1}$ inversion of gravity data using truncated unbiased predictive risk estimator for regularization parameter estimation," Geophys. J. Int., vol. 210, no. 3, pp. 1872-1887, Jun. 2017.

[26] L. Shi, H. Zhao, Y. Zakharov, B. Chen and Y. Yang, "Variable StepSize Widely Linear Complex-Valued Affine Projection Algorithm and Performance Analys," IEEE Trans. Signal Process., vol. 68, pp. 59405953, Oct. 2020.

[27] Y. Zhou, S. C. Chan, and K. L. Ho, "New sequential partial-update least mean M-estimate algorithms for robust adaptive system identification in impulsive noise," IEEE Trans. Ind. Electron., vol. 58, no. 9, pp. 44554470, Sep. 2011.

[28] S. M. Kuo, H. Chuang, and P. P. Mallela, "Integrated automotive signal processing and audio system," IEEE Trans. Consum. Electron., vol. 39, no. 3, pp. 522-532, Aug. 1993

[29] K. Doğançay and O. Tanrikulu, "Adaptive filtering algorithms with selective partial update," IEEE Trans. Circuits Syst. II, Analog Digit. Signal Process., vol. 48, no. 8, pp. 762-769, Aug. 2001.

[30] S. Zhang and W. X. Zheng, "Recursive adaptive sparse exponential functional link neural network for nonlinear AEC in impulsive noise environment," IEEE Trans. Neural Netw. Learn. Syst., vol. 29, no. 9, pp. 4314-4323, Sep. 2018.

[31] D. L. Duttweiler, "A twelve-channel digital echo canceler," IEEE Trans. Commun., vol. COM-26, no. 5, pp. 647-653, May 1978. 